\title{
Ist Telemedizin beim diabetischen Fuß riskant?
}

\author{
Welchen Nutzen hat eine telemedizinische Überwachung diabetischer Fußulzera? In einer \\ Studie heilten die Geschwüre gut ab - allerdings war auch die Mortalität deutlich erhöht.
}

\begin{abstract}
_ Für eine dänische Studie wurden 736 Patienten mit diabetischen Fußulzera in den Jahren 2010-2014 gescreent. Nach Auswahl und Randomisierung erhielten 193 Patienten zwei telemedizinische Visiten durch spezialisierte Krankenschwestern, die dem Arzt ein Foto des Ulkus und ggf. eine Wundbeschreibung übermittelten. Erst beim dritten Termin kamen die Patienten wieder in der Fußambulanz. Die 181 Kontrollpatienten stellten sich dagegen dreimal persönlich in der Ambulanz vor. Dieses Schema wurden bis zur Abheilung des Ulkus wiederholt.

Studienendpunkte waren Ulkusabheilung, Amputation oder Tod, wobei die Patienten maximal ein Jahr in der Studie blieben. Die mediane Studienzeit betrug in der Telemedizin-Gruppe 74 und in der Kontrollgruppe 91 Tage. Die mediane Zahl der telemedizinischen $\mathrm{Vi}$ -
\end{abstract}

siten betrug 3,0. In beiden Gruppen lag das mittlere Alter bei 67 Jahren, die mittlere Diabetesdauer bei 14 Jahren. Bei $72 \%$ der Patienten heilten die Ulzera komplett $a b$, ohne Unterschiede zwischen beiden Gruppen. Auch die Zahl der Amputationen unterschied sich nicht signifikant: 21 (11\%) Amputationen in der Telemedizingruppe und 26 (14\%) in der Kontrollgruppe.

Bezüglich der Mortalität fand sich jedoch ein hochsignifikanter Unterschied. Von den 9 Todesfällen traten 8 in der Telemedizingruppe auf. Nach Adjustierung für Alter, Geschlecht und Wohnort betrug die Hazard Ratio 8,68 ( $\mathrm{p}=$ $0,0001)$. Alle Verstorbenen hatten eine chronische Herzerkrankung, vier eine chronische Niereninsuffizienz und einer ein Prostatakarzinom. Sechs Patienten wiesen eine Sepsis auf, fünf eine Pneumonie und einer eine Gangrän.
Die Autoren sehen die erhöhte Mortalität als sehr kritisch an. Aufgrund der häufig erheblichen Komorbidität plädieren sie für eine besonders engmaschige Überwachung, womit eine Krankenschwester überfordert sein könnte.

- Rasmussen BSB, Froekjaer J, Bjerregaard J et al. A Randomized Controlled Trial Comparing Telemedical and Standard Outpatient Monitoring of Diabetic Foot Ulcers. Diabetes Care. 2015 38:1723-9

\section{KOMMENTAR}

Die Betreuung diabetischer Fußulzera ist sicherlich komplexer ist als jene sonstiger Wunden. Dies liegt neben den unterschiedlichen Ursachen für die Ulzera - z. B. Neuropathie, pAVK, Infektion - auch an der hohen Komorbidität der Patienten. Möglicherweise ist die persönliche Betreuung in Fußambulanzen, bei denen der "ganze" Patient gesehen wird, für die Prognose günstiger - insbesondere bei sehr kranken Patienten.

Prof. Dr. med. M. Spraul

\section{Silbernes Zeichen in der Toilette}

Eine Frau berichtete über Gewichtsabnahme, Ikterus und silberfarbene Stühle (Abb.). Der Wert des Tumormarkers CA 19-9 war erhöht, im CT des Abdomens fand sich ein Hinweis auf einen pankreatobiliären Tumor.

Silberfarbene Stühle, bekannt auch als Thomas-Zeichen, sollen pathognomonisch sein für ein Karzinom der Papilla vateri. Die metallische Farbe entsteht durch eine Mischung aus Teerstuhl und cholestatischem Stuhl. Ampullenkarzinome machen nur 0,2\% aller gastrointestinalen Karzinome aus. Obwohl silberfarbene Stühle als klassisches Zeichen gelten, sind die Daten dazu spärlich. Gelegentlich wurde sogar behauptet, dass die Prognose des Tumors davon abhängt, wie früh man diese Stuhlveränderungen erkennt und entsprechende Konsequenzen daraus zieht. Der erste Hinweis in der Literatur stammt von H. Ogilvie. Dieser Autor hat das Phänomen 1955 erstmals beschrieben, es aber in selbstloser Weise nach einem ihm bekannten Pathologen benannt, der seines Wissens nach früher bereits darauf hingewiesen hat, dass Patienten mit Papillenkarzinomen manchmal Stuhl in der Farbe von oxidiertem Silber oder von Aluminium produzieren. So nobel waren damals noch die Bräuche.

Prof. Dr. med. H. S. Füeßl

- Wagar A et al. (alibinwaqar@gmail.com). Thomas's sign. BMJ. 2016;352:11213

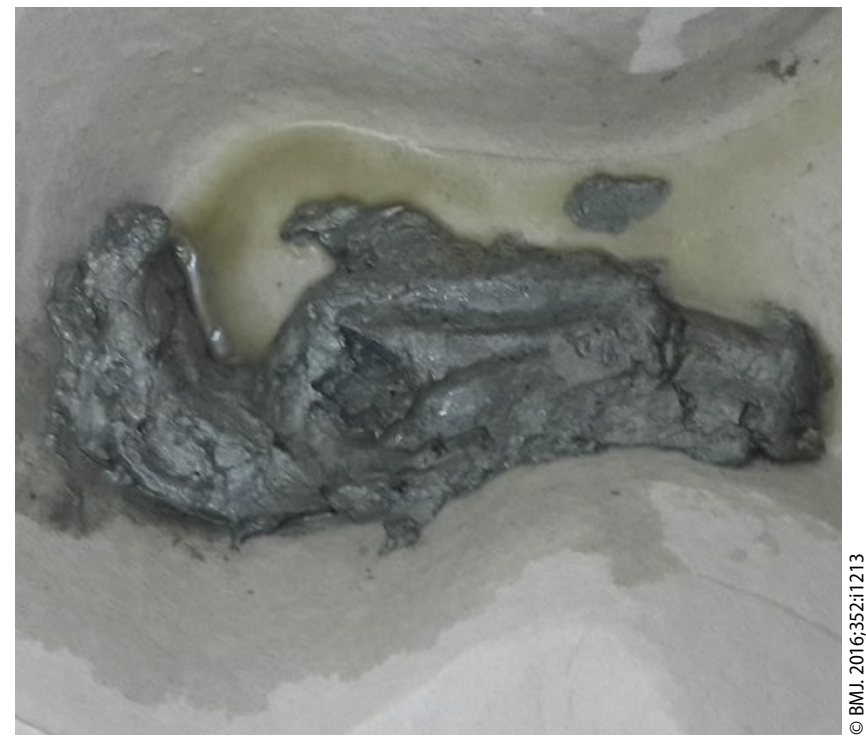

Silbern eingefärbter Stuhl. 